\title{
Structure and morphology of thin MgO films on Mo(001)
}

\author{
S. Benedetti, P. Torelli, S. Valeri \\ CNR-INFM National Research Center on nanoStructures and bioSystems at Surfaces $\left(S^{3}\right)$ and \\ Dipartimento di Fisica, Università di Modena e Reggio Emilia, Via G. Campi 213/a, I-41100 \\ Modena, Italy
}

H.M. Benia, N. Nilius

Fritz-Haber-Institut der Max-Planck-Gesellschaft, Faradayweg 4-6, D-14195 Berlin, Germany

G. Renaud

CEA, Inac, SP2M, NRS, F-38054 Grenoble, France.

\begin{abstract}
We report on the structural evolution versus thickness of $\mathrm{MgO}$ thin films grown epitaxially on $\mathrm{Mo}(001)$ and on the correlation between structure and surface morphology. The misfit strain induced by the mismatch with the substrate is relieved between 1 and $7 \mathrm{ML} \mathrm{MgO}$ due to the formation of an ordered network of interfacial misfit dislocations aligned along the $\mathrm{MgO}<110>$ directions, particularly evident after annealing the film at $1070 \mathrm{~K}$. A dislocation periodicity of about $60 \AA$ has been determined by means of Grazing Incidence X-ray Diffraction (GIXD). The dislocations induce a tilting of the surface that appears in electron diffraction along the $<100>\mathrm{MgO}$ directions for thin films and changes to $<110>$ directions when the oxide thickness increases. Scanning Tunnelling Microscopy (STM) shows the presence of a regular pattern on the surface below $7 \mathrm{ML}$ thickness associated to the dislocation network. With increasing thickness screw dislocations with non-polar steps appear on the oxide surface.
\end{abstract}




\section{INTRODUCTION}

Strain relaxation is a fundamental issue to determine the structural and morphological stability of lattice-mismatched epitaxial systems. For semiconductor or metal heteroepitaxy with a lattice mismatch with the substrate $m<10 \%$ (defined as $m=\left(a_{f}-a_{s}\right) / a_{s}$, being $a_{f}, a_{s}$ the film and substrate lattice parameters), overlayers relax the misfit strain in different ways once a certain critical thickness is exceeded. Typically relaxation occurs by: (i) formation of dislocation-free islands on top of a wetting layer (or Stranski-Krastanov growth mode), typical for semiconductor heteroepitaxy; ${ }^{1}$ or (ii) insertion of misfit dislocations in the film or islands. ${ }^{2}$ These processes depend on the balance of various energetic terms, like surface and interface energies, the energy cost for dislocation formation, as well as on kinetic limitations. These mechanisms have been investigated extensively for semiconductors and metals, while few works give detailed insights into the relaxation of thin oxide films grown on metallic surfaces. ${ }^{3,4}$

$\mathrm{MgO}$ is a suitable model oxide to study strain relief, because of its stable and inert character and its simple rocksalt structure. In addition, $\mathrm{MgO}$ thin films are used as support in heterogeneous catalysis and in magneto-electronic devices and a good understanding of their relaxation behaviour is therefore required to improve such applications. ${ }^{5,6,7}$ For this reason, $\mathrm{MgO}$ has been prepared and investigated in the form of thin films on a variety of metal substrates, such as $\operatorname{Ag}(001),{ }^{8,9,10}$ $\mathrm{Fe}(001),{ }^{11,12}$ and $\mathrm{Mo}(001) .{ }^{13,14}$ Particularly interesting is the use of $\mathrm{Mo}(001)$, because of its high thermal stability and the relatively small mismatch of $-5.2 \%$ with bulk $\mathrm{MgO}$, that allows an epitaxial growth with the relationship $\mathrm{MgO}(001) / / \mathrm{Mo}(001)$ and $\mathrm{MgO}[110] / / \mathrm{Mo}[100]$. In a recent work we have studied the morphology of $\mathrm{MgO}$ thin films deposited on $\mathrm{Mo}(001)$, where after annealing at $1070 \mathrm{~K}$ a flat and well-ordered surface is produced. ${ }^{15}$ For low oxide thickness we observed the formation of a square network with a periodicity of about $55 \AA$ and a [110] orientation in the STM, while LEED revealed the presence of surface regions with a tilt along the [100] direction with respect to the (001) plane. Similar networks have been observed on other rocksalt systems by means of STM and X-ray scattering, not only on metal-oxide systems, ${ }^{16,17}$ but also on semiconductors. ${ }^{18}$ Those experiments demonstrated the close connection between relaxation processes in the film material and the formation of defect networks. The $\mathrm{CoO} / \mathrm{Ag}(001)$ system exhibits a [110] dislocation network in x-ray diffraction. ${ }^{16}$ For $\mathrm{MgO}$ on $\mathrm{Fe}(001)$ and $\mathrm{Ag}(001)$, the appearance of a surface tilting in LEED parallel to the [100] direction is attributed to the formation of [100] dislocations by the glide system $1 / 2[110](110))^{3,4,12}$ The long range order of the dislocation network is however not discussed in those papers. Additionally the lattice mismatch with the $\mathrm{MgO}$ is rather small for substrates like $\mathrm{Ag}(-3.2 \%)$ and $\mathrm{Fe}(+3.8 \%)$, compared to the present case. Only for $\mathrm{NiO} / \mathrm{Pd}(001)$, both the square network and the tilting have been observed. ${ }^{19}$ While the first has 
been tentatively assigned to Moiré-type interference or to an ordered dislocation network, the latter has been related to the alignment of island borders.

This work aims for a detailed study of the structural evolution of the $\mathrm{MgO} / \mathrm{Mo}$ system by means of diffraction techniques and STM to analyze the correlation between relaxation behaviour and surface morphology in thin rocksalt oxide films. As Mo as refractory metal withstands high temperatures, the effect of a thorough annealing on the morphology of the metal-oxide system is investigated, too.

\section{EXPERIMENTAL}

The structure of the $\mathrm{MgO} / \mathrm{Mo}(001)$ system has been investigated by means of GIXD, Low Energy Electron Diffraction (LEED) and Primary-beam Diffraction Modulated Electron Emission (PDMEE). GIXD measurements have been performed at ESRF in Grenoble at the beamline BM32, ${ }^{20}$ using a photon energy of $18 \mathrm{keV}$. The fixed incidence angle, $\alpha_{i}=0.166^{\circ}$, was chosen below the critical angle for total external reflection in $\mathrm{Mo}\left(0.19^{\circ}\right)$, but above that of $\mathrm{MgO}\left(0.12^{\circ}\right)$. The GIXD scans shown in this paper are represented in reciprocal lattice unit (r.l.u.) scale for $h, k$ and $l$ of the reciprocal $\mathrm{Mo}(001)$ lattice, where the lattice parameter is $a=3.147 \AA$. The $l$ coordinate is perpendicular to the $\mathrm{MgO} / \mathrm{Mo}(001)$ interface.

LEED analysis was performed using a three-grid apparatus and the diffraction patterns were recorded by a CCD camera. PDMEE measurements were performed at the SESAMO laboratory in Modena in an UHV chamber with a cylindrical mirror analyzer operating in the first derivative mode $(0.6 \%$ resolution, $15 \mathrm{eV}$ modulation) and a coaxial electron gun working at $5 \mathrm{keV}$ and $1-2$ $\mu \mathrm{A} .^{21}$

STM measurements were carried out at the Fritz-Haber-Institut in Berlin with a beetle-type STM operated at liquid-nitrogen temperature $(70 \mathrm{~K})$ and ultra-high vacuum conditions $\left(\mathrm{p} \sim 5 \times 10^{-10} \mathrm{mbar}\right)$. The Mo substrate was prepared either by sputtering at $1170 \mathrm{~K}\left(\mathrm{Ar}^{+}, 600 \mathrm{eV}\right)$ or by flashing at 2300 $\mathrm{K}$, because of the different possibilities in the used machines. The $\mathrm{MgO}$ film was grown by reactive deposition of $\mathrm{Mg}$ in $\mathrm{O}_{2}$ partial pressure of $1 \times 10^{-7}$ mbar keeping the substrate at room temperature $(\mathrm{MgO}$ deposition rate $=1.22 \mathrm{ML} / \mathrm{min})$. After the deposition the samples were annealed at $1070 \mathrm{~K}$ for $10 \mathrm{~min}$.

Film thickness in this paper has been estimated by means of a quartz microbalance and XPS. In a previous paper, measurements by STM on the same system underestimated the thickness by a factor $2^{15}$ 


\section{RESULTS AND DISCUSSION}

\section{A. Film relaxation}

The structural relaxation of the $\mathrm{MgO}$ film has been studied combining different techniques to determine both the evolution of in-plane and out-of-plane lattice parameters. In-plane parameters have been determined by GIXD. In the GIXD measurements, two components in the Mo peak are recognisable that are assigned to the presence of grains in the metal substrate. Due to the preparation via high-temperature sputtering, the grain structure and therefore the shape of the Mo peak slightly varies for different experimental runs. Figure 1(a) shows the evolution of $(h, 0,0.03)$ scan along the (200) Mo Bragg peak for increasing MgO thickness. Besides the Mo peak at $h=2$ r.l.u., for $2 \mathrm{ML}$ deposit a shoulder appears at about $h=2.05$ r.l.u., that is assigned to the $\mathrm{MgO} \mathrm{Bragg}$ peak. The shoulder evolves to a well defined peak that moves to a larger $h$ value up to $2.11 \mathrm{r}$.1.u. for $25 \mathrm{ML}$ thick films, indicating the in-plane relaxation of $\mathrm{MgO}$. The in-plane lattice parameter of the oxide film is obtained from the reciprocal space position of the peaks in Fig. 1(a) fitted with a set of Gaussian peaks and it is reported in Fig. 1(b) (empty dots). The film starts relaxing at 2 ML, but even at $25 \mathrm{ML}$ it is not fully relaxed and $0.5 \%$ of the strain remains (the strain is defined here as $S=\left(d_{/ /}-d_{0}\right) / d_{0}$, where $d_{/ /}, d_{0}$ are the in-plane and the bulk Mg-O distances, respectively). This is due to the finite film thickness that prevents complete relaxation. The effect of film annealing on the release of the strain has also been studied and the evolution of the in-plane parameter as a function of thickness in annealed films is reported in Fig. 1(b) (solid markers). After thermal treatment at $1070 \mathrm{~K}$ the $1 \mathrm{ML}$ film has decreased its strain from $5.5 \%$ to $1.5 \%$, a process that is nearly saturated already at 7 ML thickness.

The out-of-plane lattice parameter has been determined with PDMEE. PDMEE plots are reported in Fig. 2(a), where the Intensity Angular Distribution (IAD) of the $\mathrm{O}$ and Mg KLL Auger signals as a function of incidence angle along the [100] Mo azimuth are shown for 2 to $28 \mathrm{ML}$ thick MgO films after annealing, together with the Mo MNN IAD of the clean substrate. The main features of the rocksalt (001) structure are evident and rationalized by the model structure in the inset. Forward focusing peaks occur at $0^{\circ}$ and $55^{\circ}$ for $\mathrm{MgO}$, corresponding to the primary beam alignment along the [001] and [111] atomic chains, respectively. Other features are mainly generated by higher order interference and cannot directly be related to specific atomic alignments, though characteristic of the $\mathrm{MgO}$ structure, as deduced from the IAD of a bulk MgO. On the other hand, Mo MNN IAD shows main features at $0^{\circ}$ and $45^{\circ}$, corresponding to the alignment along the [001] and [101] atomic chains of a bcc (001) crystal rotated in plane by $45^{\circ}$ with respect to the $\mathrm{MgO}$ structure. This confirms the expected epitaxial growth, with the $\mathrm{MgO}$ [110] direction aligned to the $\mathrm{Mo}[100]$ one. 
The IADs at low thickness are characterized by the [111] peak shifted to higher angles, indicating a tetragonal distortion of the $\mathrm{MgO}$ film as induced by the larger in-plane lattice parameter of the substrate. With relaxation of the strain at increasing thickness, the peak moves towards its bulk position. In Figure 2(b), the angular position of the [111] peak is reported as a function of film thickness for both the as-grown and the annealed films. The peak position changes not only with increasing thickness but also with annealing, suggesting that the thermal treatment improves the crystal quality and accelerates film relaxation, as already revealed from the GIXD data (Fig. 1(b)). Out-of-plane parameter $d_{\perp}$ has been extracted combining the angular position $\vartheta$ of the [111] peak obtained by PDMEE and the in-plane parameter $d_{/ /}$from GIXD, given that $\tan \vartheta=d_{/ /} / d_{\perp}$. The Mg-O distances are summarized in Fig. 2(c) as a function of the in-plane value. The solid line describes the expected behaviour from the elastic theory approximation, ${ }^{22}$ according to the equation

$$
d_{\perp}=-\gamma \cdot d_{/ /}+(1+\gamma) d_{0}
$$

where $\gamma$ is defined as $\gamma=2 C_{12} / C_{11}$ with $C_{11}, C_{12}$ the elastic stiffness constants of bulk MgO. ${ }^{23}$ The open triangle represents the unstrained bulk $\operatorname{MgO}\left(d_{\perp}=d_{/ /}=d_{0}=2.11 \AA\right)$, while $d_{/ /}=2.23 \AA$ and $d_{\perp}=2.04 \AA$ would be expected in case of perfect pseudomorphism. If we fit the experimental data for the as grown film with Eq. (1), we obtain a $\gamma$ value of 3.8, larger than the value of 0.64 obtained on a bulk $\mathrm{MgO}$. We therefore conclude that the film elastic behaviour does not correspond to the bulk one, in contrast to the behaviour of $\mathrm{NiO}$ and $\mathrm{MgO}$ films on $\mathrm{Ag}(001) .{ }^{24,25}$ In the present case, the out-of-plane compression is larger than expected, suggesting a variation in the elastic properties of the $\mathrm{MgO}$ film. This can be ascribed to the large negative mismatch with the Mo substrate, that induces a strong $\mathrm{MgO}$ deformation. The subsequent formation of interfacial dislocations modifies the relaxation characteristics, as shown below. In addition, Mo in contrast to $\mathrm{Ag}$ does not accommodate misfit itself, because of its larger hardness and its bec crystal structure as compared to rocksalt $\mathrm{MgO}$ and fec $\mathrm{Ag}$.

\section{B. Surface deformation}

During the relaxation of the film, a surface deformation becomes evident in the $\mathrm{MgO} / \mathrm{Mo}$ system. LEED spots are in fact characterized by a four-fold splitting along the $<100>\mathrm{MgO}$ directions (Fig. 3a-c) that depends linearly on the scattering vector. This is compatible with the formation of tilted regions on the surface, as it was shown in a previous work. ${ }^{15}$ The spot splitting is shown in Fig. 3e as a function of thickness and temperature. The splitting decreases with increasing thickness and temperature, indicating the progressive flattening of the tilted surface areas due to relaxation, in accordance with the observations on $\mathrm{MgO} / \mathrm{Fe}(001)$ and $\mathrm{MgO} / \mathrm{Ag}(001){ }^{3,4}$ Furthermore, with 
increasing thickness an additional broadening appears along the $<110>\mathrm{MgO}$ directions, particularly evident above $10 \mathrm{ML}$ thickness (Fig. 3c). This broadening is linearly dependent on the scattering vector and therefore related to an additional tilt rotated by $45^{\circ}$ with respect to the low-thickness behavior. However, as the spots are simply broad and not split into distinct satellites, it is much more difficult to assign them to a precise tilting angle. When the [100] tilt disappears above $15 \mathrm{ML}$ in the LEED spots, only the [110] broadening remains visible (Fig. 3d).

The hypothesis of tilted surface planes is further supported by the Full-Width-at-Half-Maximum (FWHM) and the anisotropy of peaks in the PDMEE scans. The anisotropy is hereby defined as $A=2\left(I_{\max }-I_{\min }\right) /\left(I_{\max }+I_{\min }\right)$ with $I_{\max }\left(I_{\min }\right)$ the maximum (minimum) intensity of the PDMEE peak. While anisotropy increases, FWHM decreases with increasing crystal quality and thickness of the film, as more and more scatterers contribute to the signal along the surface normal $\left(0^{\circ}\right)$. An additional contribution to the FWHM possibly comes from tilted surface regions, which add components to the main peaks that are shifted by their tilting angle with respect to the normal. If the tilting angle is small and peaks are broad, the two components cannot be resolved and only a general broadening of the peak becomes visible, while the anisotropy remains unchanged. In Fig. 4, we have shown the anisotropy (Fig. 4(a)) and the FWHM (Fig. 4(b)) of the $0^{\circ}$ peak from PDMEE scans of $\mathrm{Mg} \mathrm{KLL}$ along the $\mathrm{MgO}[100]$ azimuth. They are compared to results taken for the $\mathrm{MgO} / \mathrm{Ag}(001)$ reference system, ${ }^{26}$ where the formation of tilted regions occurs at higher thickness, with lower average tilting angles and reduced spatial order, ${ }^{4}$ due to the smaller lattice mismatch as compared to $\mathrm{MgO} / \mathrm{Mo}$. In both systems the FWHM clearly decreases while anisotropy increases with increasing $\mathrm{MgO}$ thickness, indicating the expected improvement of the crystalline quality. Before annealing, the FWHM for $\mathrm{MgO} / \mathrm{Mo}$ is systematically larger than for $\mathrm{MgO} / \mathrm{Ag}$ and a poorer order is deduced from the peak anisotropy (not shown here). The annealing decreases the FWHM of the $\mathrm{MgO} / \mathrm{Mo}$ system. However, between 2 and $10 \mathrm{ML}$ the FWHM in PDMEE IADs is larger for the $\mathrm{MgO} / \mathrm{Mo}$ peak than for $\mathrm{MgO} / \mathrm{Ag}$, although the $0^{\circ}$ peak anisotropy remains comparable. The difference in the FWHM can not therefore be explained by a reduced crystalline order, but provides a hint for the presence of tilted planes. For a $6 \mathrm{ML} \mathrm{MgO}$ film on $\mathrm{Ag}$ the FWHM is $7.6^{\circ}$. If we assume this value as the value for a flat film, the measured FWHM of $10.4^{\circ}$ for $6 \mathrm{ML} \mathrm{MgO}$ on $\mathrm{Mo}(001)$ can be reproduced by adding two Gaussian peaks shifted by $3^{\circ}$ to the fundamental peak to account for tilted areas (Fig. 4(b)). The peak broadening in the $\mathrm{MgO} / \mathrm{Mo}$ system can thus be taken as evidence for the formation of tilted regions on the surface.

In addition, pronounced differences are evident in the FWHM values of the $0^{\circ}$ peak in PDMEE scans along the $\mathrm{MgO}$ [100] and [110] directions (Fig. 4(c)), which are not compatible with a flat film. In particular the larger width along the [100] below $12 \mathrm{ML}$ is indicative of the presence of 
tilted regions along that particular crystal orientations. This is in good agreement with the LEED spots, which show distinct satellites in the $\mathrm{MgO}[100]$ direction.

\section{Misfit dislocations and morphological evolution}

Annealing the $\mathrm{MgO}$ film at $1070 \mathrm{~K}$ stimulates the appearance of a satellite in GIXD scans between the (200) Mo Bragg peak and the MgO peak (Fig. 5(a)), that grows in intensity with increasing oxide thickness. This satellite is weak after room-temperature deposition (Fig. 1(a)), but becomes clear after annealing even for the $1 \mathrm{ML}$ film. With increasing thickness, several orders of this satellite become visible with a relative intensity maximum at $7 \mathrm{ML}$. In fact, all radial scans show similar satellite peaks with constant periodicity around the main Mo Bragg peaks, as shown for a 15 ML MgO film in Fig. 5(a)-(e). This feature is assigned to the presence of a periodic network of interfacial misfit dislocations, as already reported for $\mathrm{CoO} / \mathrm{Ag}(001)$ and $\mathrm{Ag} / \mathrm{MgO}(001){ }^{16,17}$ Dislocations form at 1-2 ML when the strain starts to release. However, due to incomplete relaxation and a poor order of the dislocations at room temperature, the network is evident only after annealing the film to high temperatures. The contribution of the annealing temperature to the film relaxation is pointed out in Fig. 6, where in panel (a) $(h, 0,0.12)$ radial scans of a $7 \mathrm{ML} \mathrm{MgO}$ film are depicted for increasing temperature, while in panel (b) the evolution of the in-plane lattice parameter along the $\mathrm{MgO}[110]$ is reported as a function of temperature. The dislocation network appears distinctly above $870 \mathrm{~K}$ and releases $70 \%$ of the strain left after the deposition when increasing the temperature to $1070 \mathrm{~K}$.

Information on the orientation of the dislocation network can be gained by the positions of the satellite peaks. The expected satellite positions for the $\mathrm{MgO} / \mathrm{Mo}$ interface in presence of a misfit dislocation network parallel to $\mathrm{MgO}<110>$ directions are illustrated by the schematic representation of the $(h k 0)$ plane of the reciprocal lattice in Fig. 7. From the direct correspondence of number and positions of peaks in the measurements (Fig. 5) and in the model (Fig. 7) we can conclude that the misfit dislocations are aligned along the $\mathrm{MgO}[110]$ direction (equivalent to the $\mathrm{Mo}[100])$. Such orientation is expected for a coincidence lattice of a rocksalt $\mathrm{MgO}$ and a bcc Mo rotated by $45^{\circ}$ with respect to each other. The coincidence-site lattice is defined as the smallest super-lattice formed by substrate and overlayer and represents the most probable spatial distribution of misfit dislocations. The corresponding lattice constant is given by $\frac{a_{f} \cdot a_{s}}{a_{f}-a_{s}}$, where $a_{f}, a_{s}$ are the lattice parameter of the film and the substrate, respectively. The experimental periodicity of the dislocation network along the $\mathrm{MgO}[110]$ direction is determined from the positions of the GIXD satellites and was found to decrease from $68 \pm 3 \AA$ for a 2 ML film to $61 \pm 1 \AA$ for a 25 ML thick film. These values are very similar to the predictions of the coincidence lattice model (74 $\AA$ for 2 
ML, $60 \AA$ for $25 \mathrm{ML}$, using as $a_{f}$ the values reported in Fig. 1(b)) and tend to the value of $56 \AA$, obtained from the coincidence lattice model in case of relaxed $\mathrm{MgO}$ on Mo.

To get a complete description of the film relaxation, we finally investigated with STM the morphological evolution of the surface as a function of thickness. Figure 8(a-b) shows the surface of a $2 \mathrm{ML}$ film annealed at $720 \mathrm{~K}$ and $1070 \mathrm{~K}$, respectively. In the case of mild annealing the film is not completely closed and exhibits islands separated by holes. Annealing at high temperature provokes the coalescence of the islands and the formation of a continuous and flat film, disrupted only by a few domain boundaries with 10-20 nm separation between the original islands. An example for a domain boundary is marked with a dashed line in Fig. 8(b). The growth therefore follows a perfect 2D mode, as expected from the large difference in surface free energy of $\mathrm{MgO}$ $\left(1.16 \mathrm{~J} / \mathrm{m}^{2}\right)$ and Mo $\left(3.87 \mathrm{~J} / \mathrm{m}^{2}\right)$. In the range between 2 and $7 \mathrm{ML}$, a square pattern with a periodicity of $55 \pm 5 \AA$ and [110] orientation is observed on the surface (Fig. 8(b)), as already reported in Ref. 15. The square pattern covers the whole film, overgrowing even the domain boundaries in some cases. Occasionally, bright lines are inserted into the square pattern, as shown in the region marked by a rectangle in Fig. 8(b) and enlarged in Fig. 8(d).

When thickness increases above $15 \mathrm{ML}$, the square pattern disappears and an almost flat surface is obtained (Fig. 8c). Here screw dislocations, as marked by an arrow in Fig. 8(c), are evident, which form the starting point for monatomic steps along the non-polar $\mathrm{MgO}<100>$ directions. These steps are the remnants of the randomly-oriented domain boundaries in thin films, which gradually align along the $\mathrm{MgO}<100>$ direction with increasing film thickness. The alignment results from the vanishing stabilization effect of polar edges by the metal support and the better crystalline order of thicker films. ${ }^{27}$ Further thickness increase leads to the development of flat films having exclusively $<100>$ borders, while the LEED shows a simple (1x1) pattern. ${ }^{15}$

\section{Discussion}

The structural model that can be derived from the presented results is depicted in Fig. 8(e). Due to the annealing, the $-5.2 \%$ lattice mismatch with the substrate rapidly relaxes in the $\mathrm{MgO}$ film. Between 1 and $7 \mathrm{ML}$, the main part of the misfit strain is relieved by introducing [110] misfit dislocations. Since the square pattern observed by STM in this thickness range is consistent both in size and orientation with the GIXD results, we ascribe it to the interfacial dislocation network, although the underlying contrast mechanism is not clear and might be of topographic as well as of electronic origin.

The assignment of the bright lines in STM to the dislocation network is also supported by the presence of threading dislocations, as seen in Fig. 8(b) and enlarged in Fig. 8(d). Because every 
dislocation must be terminated at the free crystal surface, there are two threading dislocations that connect each misfit dislocation with the surface layer. Starting from the interfacial layer, additional atomic planes are progressively added along the $<110>$ directions with a periodicity of about $60 \AA$ to release the strain of the $\mathrm{MgO}$ film. The propagation of those dislocations towards the surface produces a periodic deformation of the film, as apparent in STM images of the oxide surface. This deformation is directly related to the strain relief in the lattice-mismatched $\mathrm{MgO} / \mathrm{Mo}$ system and responsible for the surface tilting with respect to the (001) plane, as revealed by the LEED satellites and the enlarged PDMEE peaks. Tilted surface planes are however not evident in STM and GIXD. The latter technique is indeed more bulk sensitive than electron diffraction, that hampers the detection of small surface perturbations such as a tilting of the surface. A tilting of roughly $5^{\circ}$ starts to appear in the second layer and includes large portions of the surface, as the central spot in LEED is almost completely suppressed (Fig. 3b). ${ }^{*}$ The tilt direction is parallel to the $\mathrm{MgO}[100]$ and thus rotated by $45^{\circ}$ with respect to the dislocation lines. The driving force for the misalignment of tilting and dislocation lines might be found in the strong MgO-Mo interface interaction. As the most efficient relaxation is obtained by inserting additional atomic rows along the $\mathrm{MgO}[110]$ direction (the $\mathrm{Mo}[100]$ direction), dislocation lines might preferentially align in this particular way.

In addition, the Mo-Mg and Mo-O interactions induce a variation in the atomic out-of-plane displacement across the network. Due to the relaxation, $\mathrm{O}$ and $\mathrm{Mg}$ atoms are not always in top and hollow sites, respectively, but there are regions of poor (areas with misfit dislocations) and good matching with the substrate atoms (in between the dislocation lines) (see Fig. 8(e)). In the first regions, $\mathrm{O}$ atoms sit most probably in Mo hollow sites, while $\mathrm{Mg}$ are in top positions and the $\mathrm{MgO}-$ Mo interface distance is therefore large. In regions of good matching, $\mathrm{O}$ and $\mathrm{Mg}$ atoms are in top and hollow sites, respectively, and the film is closer to the substrate. This assumption is in accordance with theoretical predictions of a larger interfacial distance when $\mathrm{Mg}$ and not $\mathrm{O}$ atoms occupy on-top sites of the metal support. ${ }^{28}$ Also experiments confirm that the O-top site is the most stable adsorption site on Ag substrates. ${ }^{29}$ This vertical displacement can here be responsible for the tilted $\mathrm{MgO}$ planes determined from the diffraction data and shown in the model (Fig. 8(e), only $\mathrm{Mg}$ atoms are represented).

The $<100>$ tilting is in accordance with previous observations on rocksalt oxides, ${ }^{3,4,12,19}$ while the orientation of the dislocation network has not been clearly determined before. As LEED does not give a direct measure of the dislocation orientation, they were often assumed to be aligned along the [100] direction, since no direct techniques were employed. ${ }^{3,4}$ Only in the case of $\mathrm{CoO} / \mathrm{Ag}$, [110]

\footnotetext{
* The suppression of the central peaks of the LEED spots might also be related to interference effects induced by the vertical displacements of region in the $\mathrm{MgO}$ super-structure cell, as depicted in Fig.8e.
} 
oriented dislocations were directly observed by GIXD, ${ }^{16}$ while for $\mathrm{NiO} / \mathrm{Pd}$ a square [110] pattern visible in STM was tentatively assigned to interfacial dislocations. ${ }^{19}$

With increasing thickness, the tilting angle decreases and almost flat and fully relaxed $\mathrm{MgO}$ surfaces with negligible influence of the interfacial dislocation network are observed above $15 \mathrm{ML}$ (Fig. 8c). In this thickness regime, screw dislocations associated with monatomic non-polar steps occur, which induce a tilting of the surface along the $<110>$ directions (Fig. 8(c)). The dislocations are widely spaced $(10-20 \mathrm{~nm})$ and show no long-range order, in contrast to the interfacial network. As a consequence, they introduce a distribution of tilting angles and cause a star-like broadening of the LEED spots in $<110>$ directions, while sharp satellites as observed for lower thickness are not observed. Following the evolution of the surface morphology, the monatomic steps seem to be related to the domain boundaries left by the island coalescence, which gradually align with the $\mathrm{MgO}$ $<100>$ for increasing thickness. In this way, the screw dislocations could be related to the lateral misfit of merging oxide islands ${ }^{30}$ or alternatively form at buried defects of the interfacial dislocation network (e.g. spiral structures nucleated on threading dislocations). ${ }^{31}$ However, they might also be introduced in a non-regular manner into the thicker film to release the remaining misfit with the Mo support. A complete analysis of the crystallographic nature of these screw dislocations cannot be performed on the basis of the present experimental results.

Screw dislocations have never been observed before in oxide films of rocksalt structure, as the large thickness at which they occur usually prevents STM experiments. Also the resulting $<110>$ tilting was not observed, with the exception of $\mathrm{CoO} / \mathrm{Ag}(001)^{16}$ and $\mathrm{NiO} / \mathrm{Pd}(001)$, where both $<100>$ and $<110>$ tilting was reported as a function of oxide thickness. ${ }^{19}$ Comparing the different oxide systems, it becomes however not clear whether the relaxation follows different mechanisms in the various oxide-metal systems or the $<110>$ tilting was missed due to: i) the thickness range investigated, ${ }^{4,12}$ ii) the low annealing temperatures or iii) the increased broadening of the LEED spots for thick films that prevents a detailed analysis. ${ }^{3}$

\section{CONCLUSIONS}

To summarize, we have reported on the investigation of the structural and morphological evolution of the $\mathrm{MgO} / \mathrm{Mo}(001)$ system. Between 1 and $7 \mathrm{ML}$ the film relaxes almost completely the misfit strain by the formation of a regular array of interfacial misfit dislocations with $60 \AA$ periodicity and $\mathrm{MgO}[110]$ orientation. The crucial role of high temperature annealing for the film relaxation has clearly been demonstrated. The misfit dislocations introduce a periodic modulation of the in-plane parameter, resulting in regions of good and poor matching with the substrate lattice. The strong interaction with the Mo substrate atoms in those different regions determines the tilting of the $\mathrm{MgO}$ 
planes along the $\mathrm{MgO}[100]$ direction that flattens once the dislocations are buried far below the oxide surface at about 15 ML.

Between 15 and $20 \mathrm{ML}$ the surface is characterized by non-polar [100] MgO steps that could be remnants of the coalescence of $\mathrm{MgO}$ islands in the first stages of growth. The steps are often pinned by screw dislocations, which help releasing the remaining strain in the film and induce a surface tilting along the $\mathrm{MgO}$ [110] direction. Complete relaxation of $\mathrm{MgO}$ films on $\mathrm{Mo}(001)$ is not observed up to 25 ML film thickness.

\section{Acknowledgments}

The authors wish to acknowledge the financial support by the EU (FP6STRP"'GSOMEN").

\section{List of figures}

Fig. 1
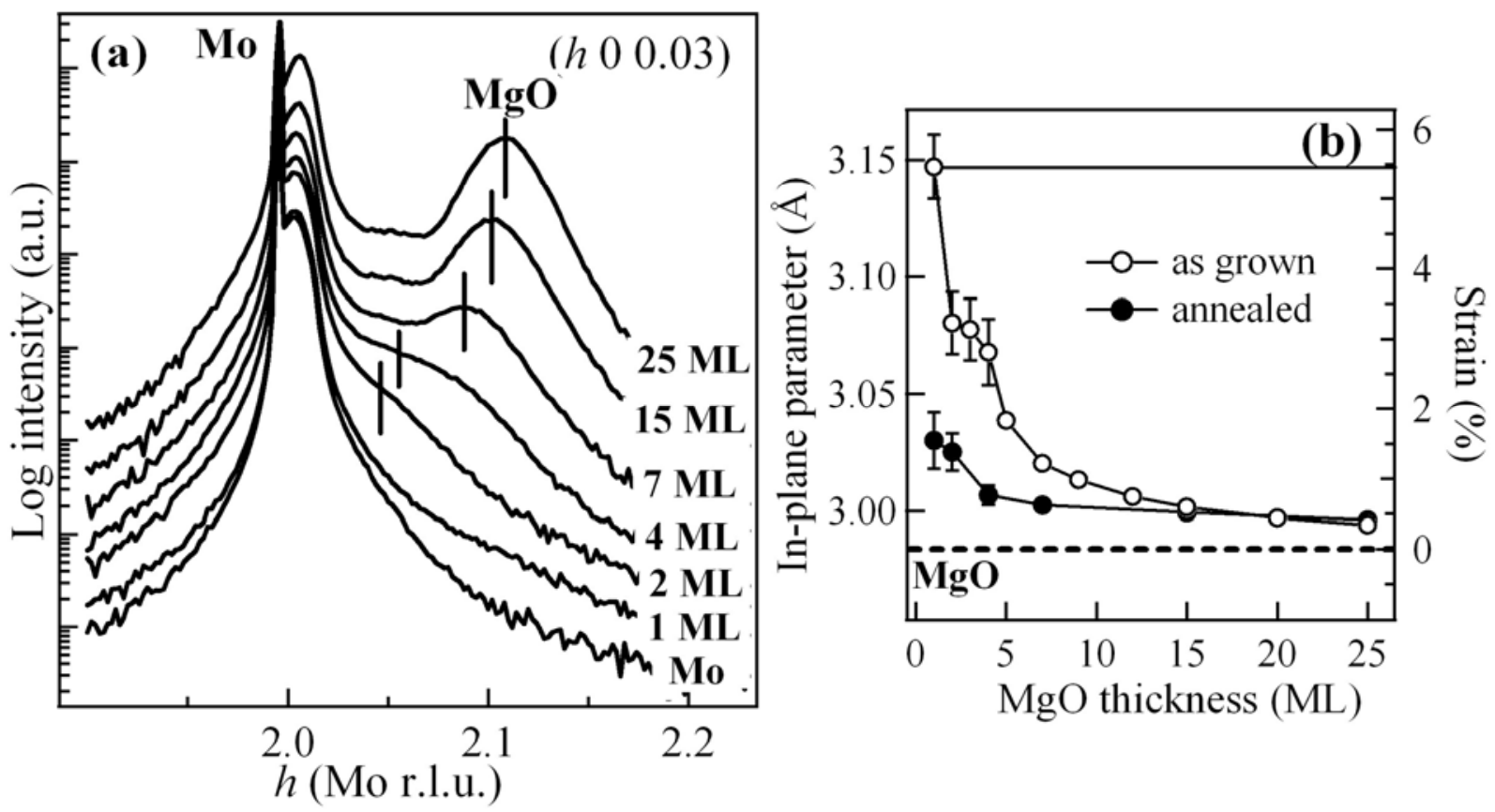

Fig. 1: (a) Radial scans along the $(h, 0,0.03)$ direction around the (200) Mo Bragg peak for as grown $\mathrm{MgO}$ film of increasing thickness. Curves have been vertically shifted for clarity. (b) Evolution of the in-plane $\mathrm{MgO}$ parameter, along the $\mathrm{MgO}[110]$ direction, as a function of film thickness before (o) and after annealing at $1070 \mathrm{~K}(\bullet)$. 
Fig. 2
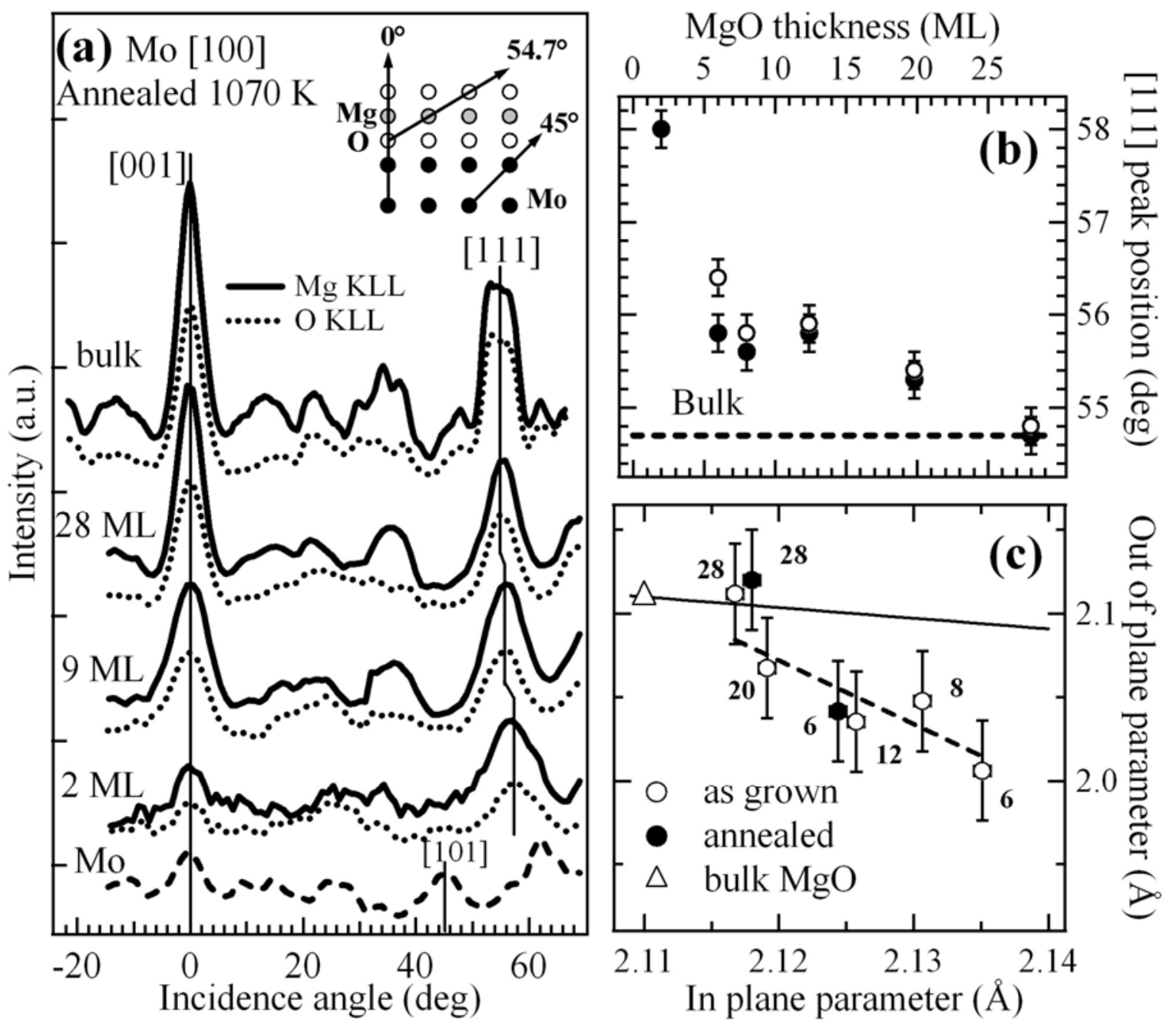

Fig. 2: (a) IADs of Mg KLL (solid line) and O KLL (dotted line) Auger signals along the [100] Mo azimuth for 2-28 ML MgO after annealing at $1070 \mathrm{~K}$. IADs of bulk MgO (top curves) and the Mo MNN IAD of the clean substrate (dashed line) are shown for comparison. Each plot has been scaled to its $0^{\circ}$ peak anisotropy. Inset: side view of the rocksalt $\mathrm{MgO}$ on the bcc Mo structure cut along the Mo [100]. (b) Angular position of the [111] peak of Mg KLL IAD along the [100]Mo azimuth as a function of $\mathrm{MgO}$ thickness for the as-grown (o) and annealed (•) samples.

(c) Out-of-plane $\mathrm{Mg}-\mathrm{O}$ distance as a function of in-plane distance obtained by PDMEE and GIXD analysis, respectively. The values for a bulk $\mathrm{MgO}$ are shown with an open triangle. The solid line represents the values calculated with the $\mathrm{MgO}$ bulk elastic constants $(\gamma=0.64$, see text for details), while the dashed line indicates the best fit of the experimental data for as grown (o) films $(\gamma=3.8)$. Numbers next to each point indicate the corresponding MgO thickness in ML. 
Fig. 3
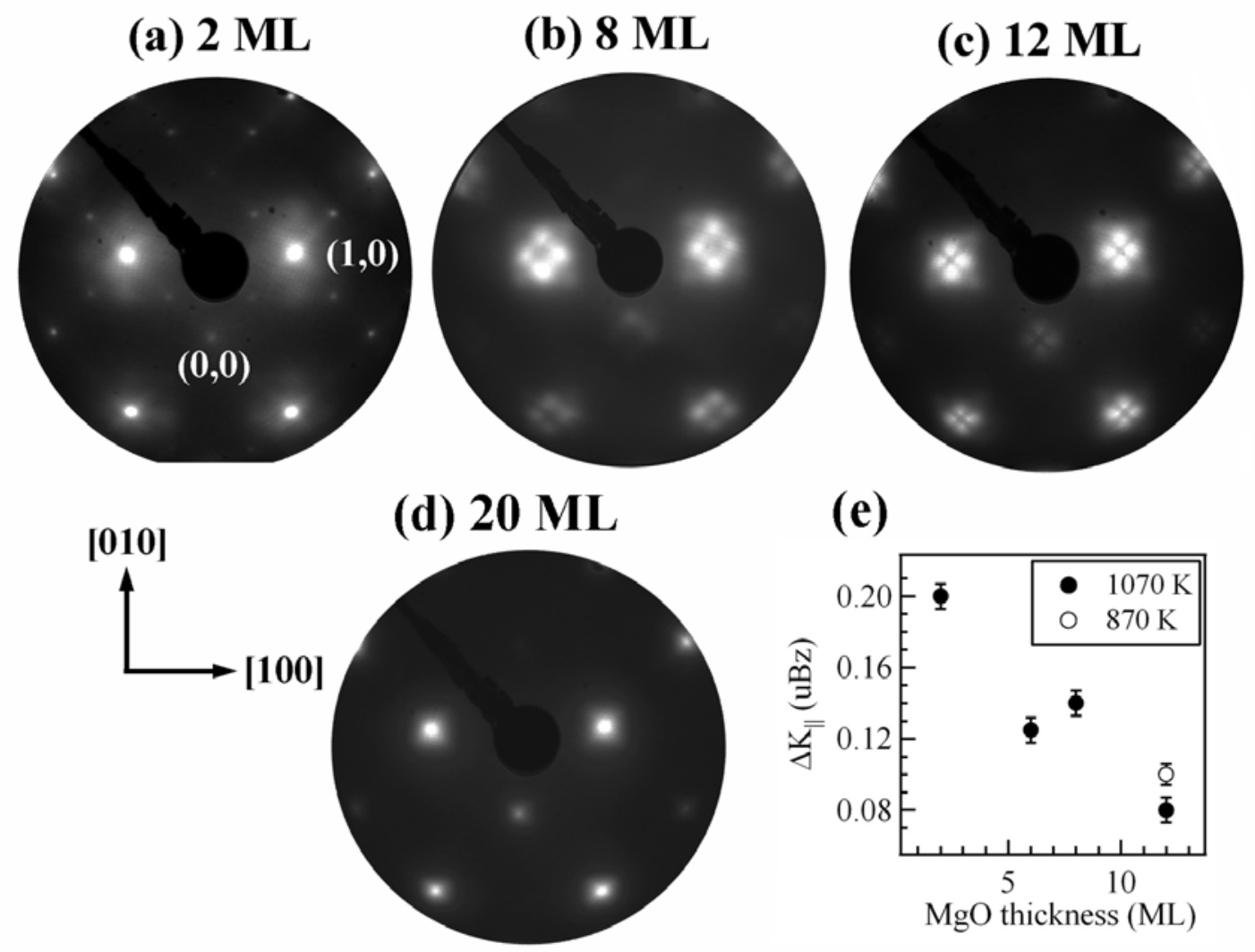

Fig. 3: LEED pattern for (a) $2 \mathrm{ML}$, (b) $8 \mathrm{ML}$, (c) $12 \mathrm{ML} \mathrm{MgO,} \mathrm{(d)} 20 \mathrm{ML}$ on Mo(001) and (e) spot splitting in units of the first Brillouin zone $(\mathrm{uBz})$ as a function of $\mathrm{MgO}$ thickness after annealing at $1070 \mathrm{~K}(\bullet)$ and for $12 \mathrm{ML}$ after annealing at $870 \mathrm{~K}(\mathrm{o})\left(\mathrm{E}_{\mathrm{p}}=100 \mathrm{eV}\right)$. 
Fig. 4
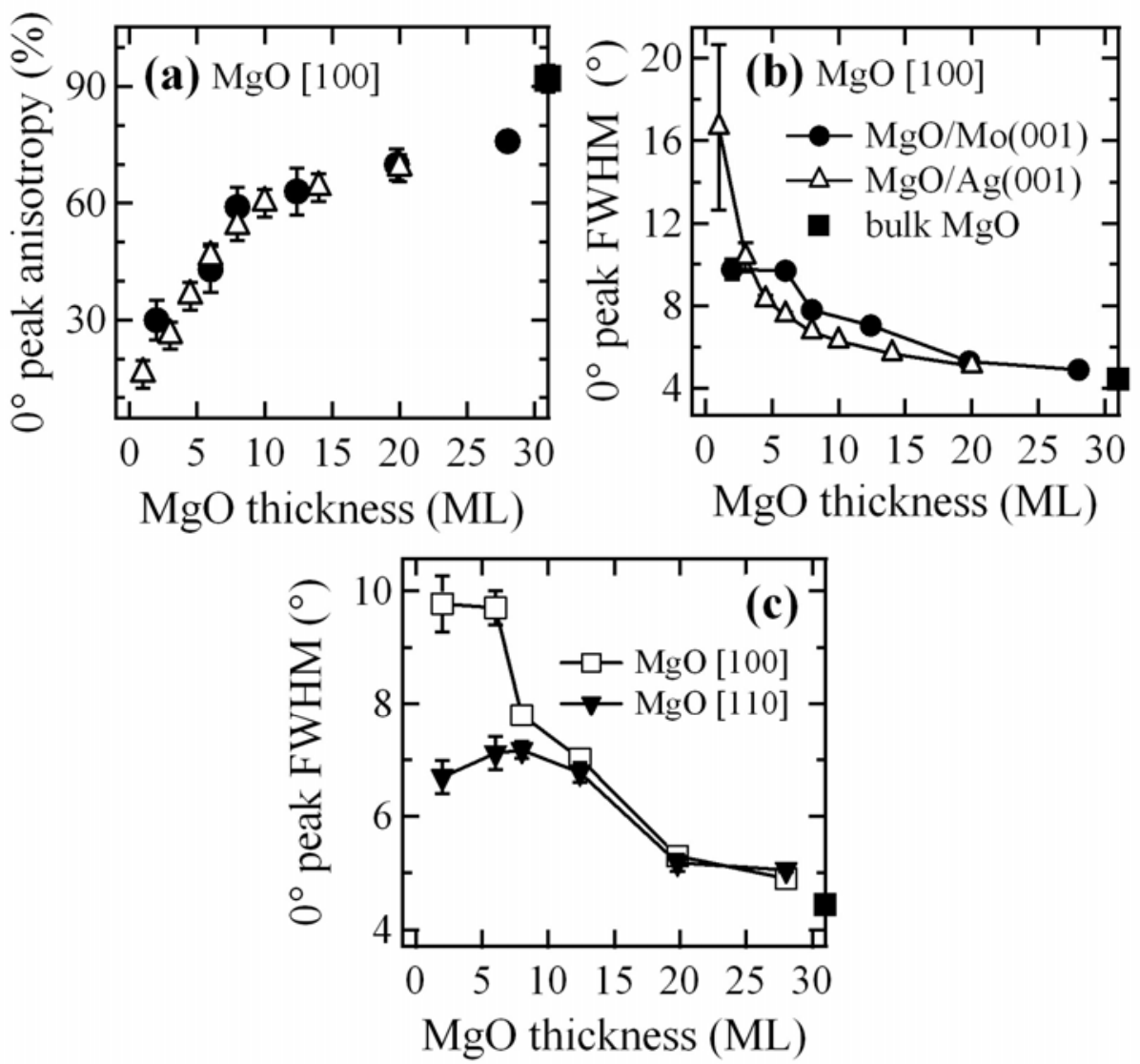

Fig. 4: (a) Anisotropy and (b) FWHM of the $0^{\circ}$ peak of Mg KLL IAD along the [100] $\mathrm{MgO}$ azimuth as a function of oxide thickness in $\mathrm{MgO} / \mathrm{Mo}(001)$ after annealing (•) and in $\mathrm{MgO} / \mathrm{Ag}(001)$ system (口). ${ }^{26}$ Bulk values are reported for comparison (a). (c) FWHM of the $0^{\circ}$ peak of Mg KLL IAD along the $\mathrm{MgO}[100](\boldsymbol{\nabla})$ and [110] azimuth ( $\square$ ). Where not reported, the marker size represents the error bar on the y-axis. 
Fig. 5
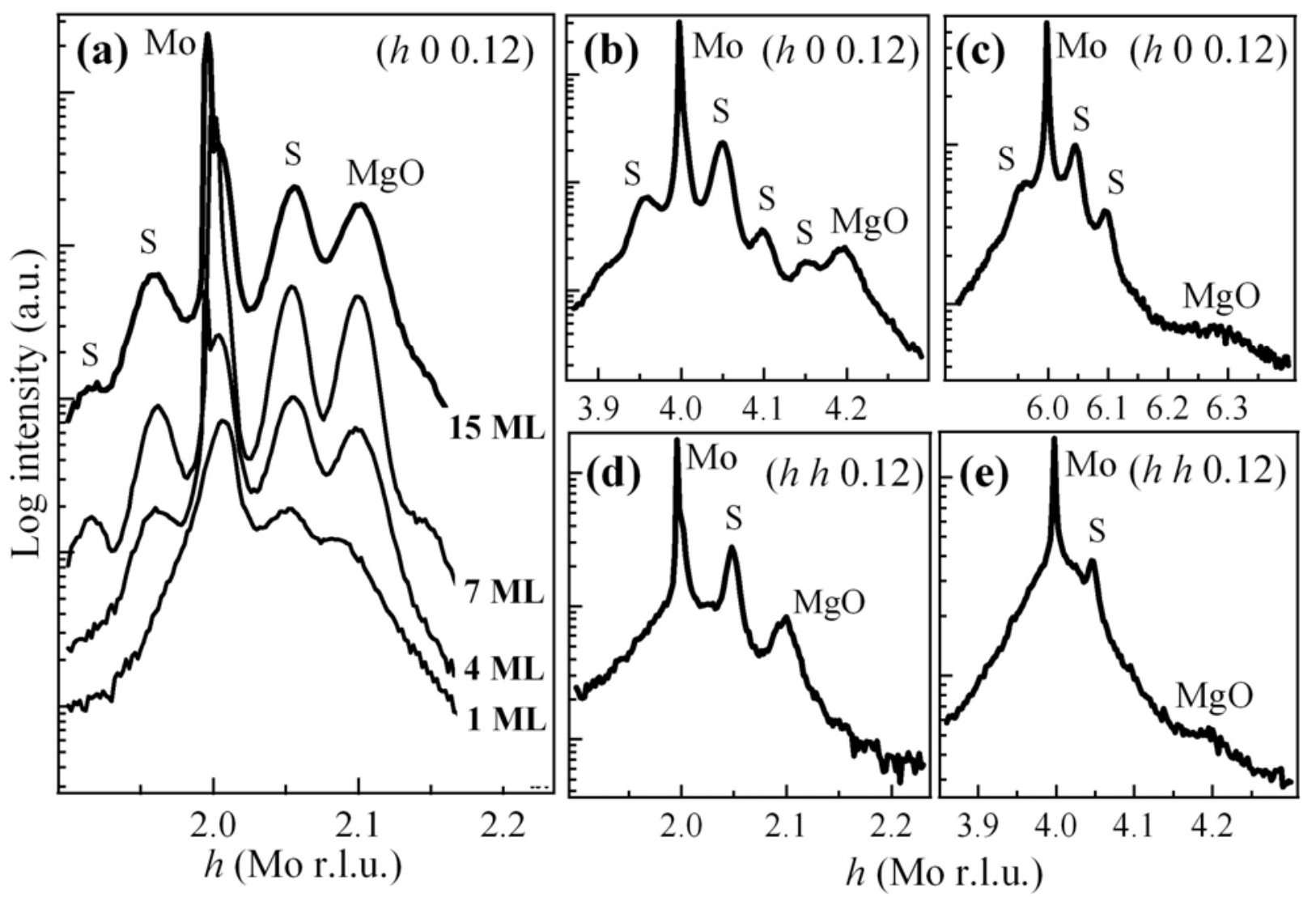

Fig. 5: (a) Radial scans along the $(h, 0,0.12)$ direction around the (200) Mo Bragg peak for $\mathrm{MgO}$ films of increasing thickness annealed at $1070 \mathrm{~K}$. (b-e) Radial scans on a $15 \mathrm{ML} \mathrm{MgO}$ film on Mo(001) annealed at $1070 \mathrm{~K}$. Diffraction satellites due to the interfacial dislocation network are indicated with a "S". The $l$ value of 0.12 has been chosen to maximize the satellite/bulk relative contributions. 
Fig. 6
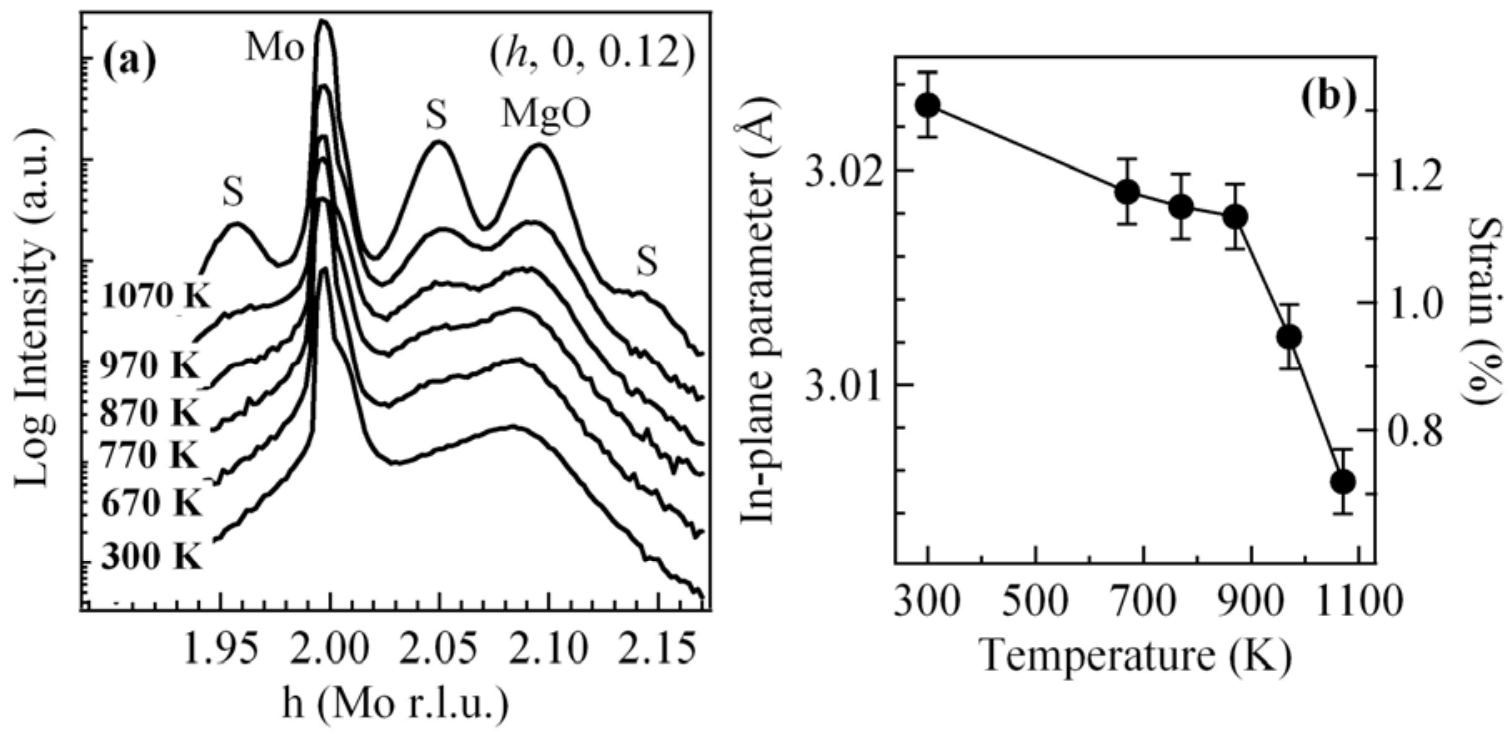

Fig. 6: (a) Radial scans along the $(h, 0,0.12)$ direction around the (200) Mo Bragg peak for a $7 \mathrm{ML}$ $\mathrm{MgO}$ film annealed at increasing temperature. (b) Evolution of the in-plane $\mathrm{MgO}$ lattice parameter along the $\mathrm{Mo}[100](\mathrm{MgO}[110])$ direction as a function of annealing temperature.

Fig. 7

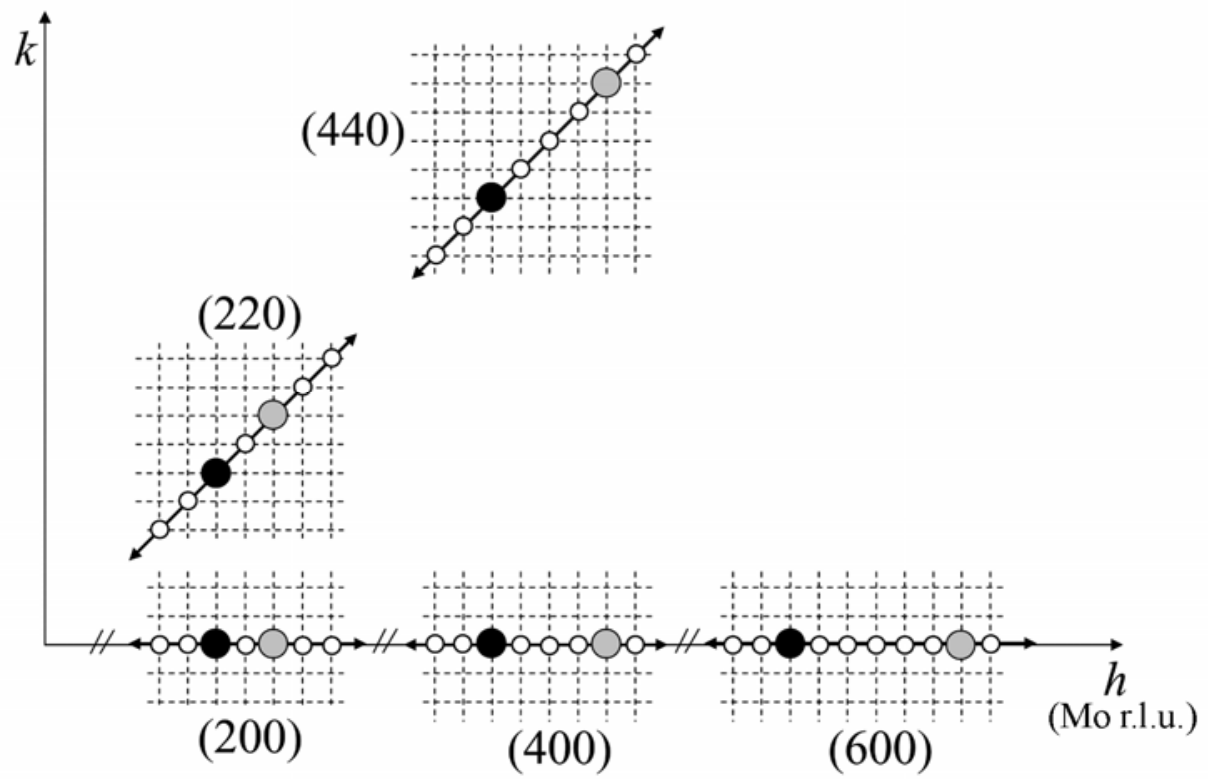

Fig. 7: Schematic representation of the $(h k 0)$ plane of the reciprocal lattice of the $\mathrm{MgO} / \mathrm{Mo}(001)$ interface with an interfacial network of misfit dislocation. Black and grey dots indicate the positions of the Mo and $\mathrm{MgO}$ Bragg peaks, respectively. The reciprocal lattice of the dislocation network is represented with dashed lines. White dots represent the location of the satellites as expected for a $[110] \mathrm{MgO}([100] \mathrm{Mo})$ dislocation network. 
Fig. 8
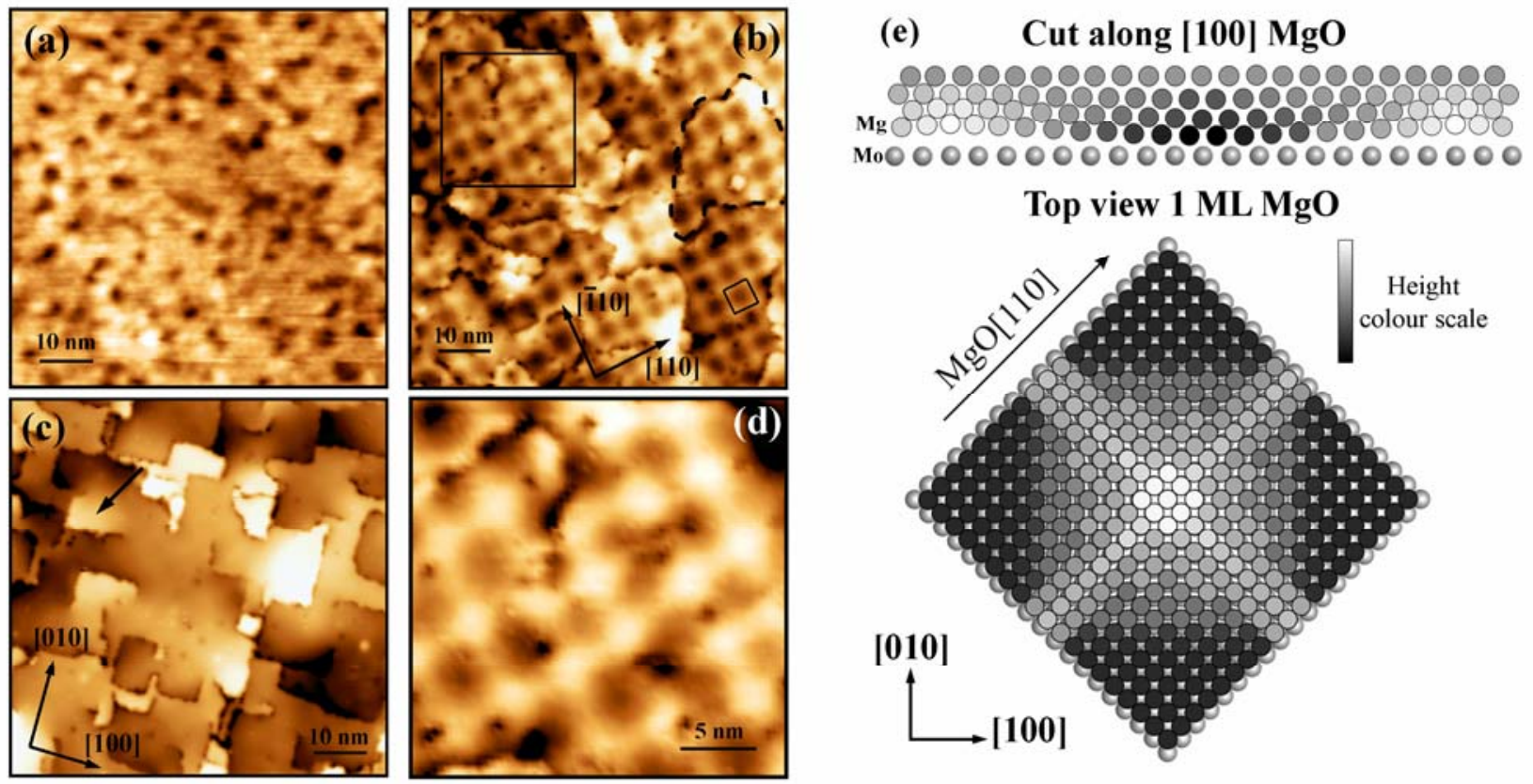

Fig. 8: $70 \times 70 \mathrm{~nm}^{2} \mathrm{STM}$ images of $\mathrm{MgO}$ films on $\mathrm{Mo}(001)$ of: (a) $2 \mathrm{ML}$ annealed at $720 \mathrm{~K}(\mathrm{U}=3.3$ $\mathrm{V})$; (b) $2 \mathrm{ML}$ annealed at $1070 \mathrm{~K}(\mathrm{U}=3.5 \mathrm{~V})$ with the unit cell of the dislocation network and a domain boundary marked with solid and dashed lines, respectively; (c) $15 \mathrm{ML}$ annealed at $1070 \mathrm{~K}$ $(\mathrm{U}=4.8 \mathrm{~V})$ with the arrow indicating a [110] tilted surface region in proximity of a screw dislocation; (d) $25 \times 25 \mathrm{~nm}^{2}$ zoom-in of the region marked in panel (b).

(e) Structure model showing a cross-section along the $\mathrm{MgO}[100]$ direction and a top view of the $\mathrm{MgO}$ super-structure cell. The model demonstrates the interrelation between the interfacial dislocation (grey lines) and the surface tilting. Only the $\mathrm{Mg}$ atoms are depicted for sake of clarity. Bright and dark areas in the model correspond to the respective regions observed in the STM images in (b) and (d). 


\section{References}

${ }^{1}$ Y.-W. Mo, D.E. Savage, B.S. Swartzentruber, M.G. Lagally, Phys. Rev. Lett. 65, 1020 (1990)

${ }^{2}$ J. W. Matthews and A. E. Blakeslee, J. Cryst. Growth 27, 118 (1974)

${ }^{3}$ M. Dynna, J.L. Vassent, A. Marty, B. Gilles, J. Appl. Phys. 80, 2650 (1996)

${ }^{4}$ J. Wollschläger, D. Erdös, H. Goldbach, R. Höpken, K.M. Schröder, Thin Solid Films 400, 1 (2001)

${ }^{5}$ M. Sterrer, T. Risse, U. Martinez Pozzoni, L. Giordano, M. Heyde, H.-P. Rust, G. Pacchioni, and H.-J. Freund, Phys. Rev. Lett. 98, 096107 (2007)

${ }^{6}$ G. Pacchioni, L.Giordano, and M.Baistrocchi, Phys. Rev. Lett. 94, 226104 (2005)

${ }^{7}$ S. Yuasa, T. Nagahame, A. Fukushima, Y. Suzuki and K. Ando, Nature Mat. 3, 868 (2004)

${ }^{8}$ S. Valeri, S. Altieri, A. di Bona, P. Luches, C. Giovanardi, and T.S. Moia, Surf. Sci. 507-510, 311 (2002)

${ }^{9}$ J. Wollschläger, J. Viernow, C. Tegenkamp, D. Erdös, K.M. Schröder, and H. Pfnür, Appl. Surf. Sci. 142, 129 (1999)

${ }^{10}$ S. Schintke, S. Messerli, M. Pivetta, F. Patthey, L. Libioulle, M. Stengel, A. De Vita, and W.D Schneider, Phys. Rev. Lett. 87, 276801 (2001)

${ }^{11}$ H.L. Meyerheim, R. Popescu, J. Kirschner, N. Jedrecy, M. Sauvage -Simkin, B. Heinrich, and R. Pinchaux, Phys. Rev. Lett. 87, 076102 (2001)

${ }^{12}$ M. Klaua, D. Ullmann, J. Barthel, W. Wulfhekel, J. Kirschner, R. Urban, T.L. Monchesky, A. Enders, J.F. Cochran, B. Heinrich, Phys. Rev. B 64, 134411 (2001)

${ }^{13}$ M. Sterrer, E. Fischbach, T. Risse, and H.-J. Freund, Phys. Rev. Lett. 94, 186101 (2005)

${ }^{14}$ M.C. Gallagher, M.S. Fyfield, L.A. Bumm, J.P. Cowin, and S.A. Joyce, Thin Solid Films 445, 90 (2003)

${ }^{15}$ S. Benedetti, H. M. Benia, N. Nilius, S. Valeri, and H. J. Freund, Chem. Phys. Lett. 430, 330 (2006)

${ }^{16}$ P. Torelli, E.A. Soares, G. Renaud, L. Gragnaniello, S. Valeri, X.X. Guo, P. Luches, Phys. Rev.

B 77, 081409(R) (2008)

${ }^{17}$ G. Renaud, P. Guénard, and A. Barbier, Phys. Rev. B 58, 7310 (1998)

${ }^{18}$ G. Springholz and K. Wiesauer, Phys. Rev. Lett. 88, 015507 (2002)

${ }^{19}$ J. Schoiswohl, W. Zheng, S. Surnev, M.G. Ramsey, G. Granozzi, S. Agnoli, F.P. Netzer, Surf. Sci. 600, 1099 (2006)

${ }^{20}$ ESRF, 6 rue Jules Horowitz, BP 220, F-38043 Grenoble Cedex, France, www.esrf.eu

${ }^{21}$ S. Valeri, A. di Bona, G.C. Gazzadi, Surf. Interf. Anal. 21, 852 (1994)

${ }^{22}$ P.M. Marcus, F. Jona, J. Phys. Chem. Solids 55, 1513 (1994)

${ }^{23}$ J. P. Hirth, J. Lothe, Theory of Dislocations (McGraw-Hill, New York, 1982)

${ }^{24}$ P. Luches, S. D’Addato, S. Valeri, E. Groppo, C. Prestipino, C. Lamberti, F. Boscherini, Phys. Rev B 69, 045412 (2004)

${ }^{25}$ E. Groppo, C. Prestipino, C. Lamberti, P. Luches, C. Giovanardi, F. Boscherini, J. Phys. Chem. B 107, 4597 (2003)

${ }^{26}$ S. Valeri, S. Altieri, A. di Bona, C. Giovanardi, T. S. Moia, Thin Solid Films 400, 16 (2001)

${ }^{27}$ A.M. Ferrari, S. Casassa, C. Pisani, S. Altieri, A. Rota, S. Valeri, Surf. Sci. 588, 160 (2005)

${ }^{28}$ L. Giordano, J. Goniakowski, G. Pacchioni, Phys. Rev. B 67, 045410 (2003)

${ }^{29}$ C. Giovanardi, A. di Bona, T.S. Moia, S. Valeri, C. Pisani, M. Sgroi, M. Busso, Surf. Sci. 505, L209 (2002)

${ }^{30}$ G. Welder, C.M. Schneider, A. Trampert, R. Koch, Phys. Rev. Lett. 93, 236101 (2004)

${ }^{31}$ G. Springholz, N. Frank, G. Bauer, Appl. Phys. Lett. 64, 2970 (1994) 